\title{
Dispersion and Fundamental Absorption Edge Analysis of Doped a-Si:H thin Films I : p-type
}

\author{
${ }^{1}$ Assem Bakry \\ Physics Dept., Faculty of Science, Ain-Shams Univ., Cairo, Egypt 11566.
}

The refractive indices and the extinction coefficients of Al doped a-Si:H films are determined from their transmittance spectrograms over a wide energy range, 0.45-2.5 eV. Analysis of the refractive index data yields the values of the long wavelength dielectric constant, the average oscillator wavelength, average oscillator strength, average oscillator energy, dispersion energy, and lattice energy. The change of the absorption edge versus photon energy shows the transition to be of the indirect type and gives as well the Urbach energy. The real and imaginary parts of dielectric constant are used to calculate the free carrier plasma resonance frequency, optical relaxation time and the ratio of free carrier concentration to the free carrier effective mass.

\section{Introduction}

The suitability of hydrogenated amorphous silicon (a-Si :H) for photovoltaic devices has stimulated great interest in the preparation and characterization of this material. Since the first a-Si:H solar cell was made by Carlson et al. [1] the technology has improved tremendously, leading to reported initial conversion efficiencies exceeding 15\% [2]. For optoelectronic devices, an accurate determination of the optical constants such as bandgap, absorption coefficient, refractive index, extinction coefficient with doping concentration and wavelength of semiconductor thin films are important to precisely model their spectral response.

The optical constants also provide information about the electronic band structure, the band tail, and localized states [3]. This practical and fundamental significance has led to a number of studies [4] concerned with the optical properties of a-Si:H and related alloys.

\footnotetext{
1 e-mail: assem_sedfy@asunet.shams.edu.eg, Phone: +202 25057102
} 
The approach of alloying a-Si:H with materials of lower coordination number was previously attempted. The di-valent O-atoms was used [5] and found to decrease the average coordination number in a pronounced matter. Alloying a-Si:H with the tri-valent $\mathrm{Al}$ was also performed [6], and found to reduce the network strain due to lowering of the average coordination number.

In this work, a comprehensive study for the optical properties of a$\mathrm{Si}: \mathrm{Al}: \mathrm{H}$ is carried out. The dispersion behavior of this structure allows the real and imaginary parts of the refractive index to be obtained. The real part of the refractive index provides values for the oscillation energy, dispersion energy and lattice energy to be quantified. The imaginary part of the refractive index yields the variation of the absorption coefficient due to the increase in $\mathrm{Al}$ concentration. The disorder in the material is traced through the evaluation of the change in the Urbach energy. The indirect transitions in the optical gap have been studied as well.

\section{Experimental procedures}

The preparation of the films was carried out in Klaushal Ziellerfield Technical University in Germany. The investigated films were prepared on Corning 7059 glass substrates at a temperature $300^{\circ} \mathrm{C}$ as follows. First, silicon was evaporated from an electron beam heated vitreous Carbon crucible at a rate of approximately $3 \mathrm{~A}^{\circ}$ per second to a thickness of about $5000 \mathrm{~A}^{\circ}$. Then the dopant material $(\mathrm{Al})$ was introduced into the material through thermally evaporating different rates. The thickness of evaporated films was determined interferometrically [7]. The base pressure of vacuum was $10^{-7} \mathrm{mbar}$. During the entire evaporation process, atomic hydrogen was blown at the growing film from a radio frequency dissociation system. The resulting hydrogen pressure in the vacuum vessel was $3 \times 10^{-5}$ mbar and the hydrogen flux through the dissociator was about $16 \mathrm{ml} \mathrm{N} / \mathrm{min}$. In this environment most dangling bonds in $\mathrm{Si}$ were saturated by hydrogen and the properties of amorphous films of pure $\mathrm{Si}$ are similar to those of a-Si:H prepared by Silane decomposition [8]. Al concentrations were estimated using Secondary Ion Mass Spectrometry (SIMS). It is an analytical technique that detects very low concentrations of dopants and impurities. It can provide elemental depth profiles over a depth range from a few angstroms to tens of microns. SIMS works by sputtering the sample surface with a beam of primary ions. Secondary ions formed during the sputtering are extracted and analyzed using a mass spectrometer. These secondary ions can range from matrix levels down to sub-parts-per-million trace levels. The concentration of dopants was found to range from 3.6-8.3 at.\%. The hydrogen content in all the used films was kept fixed at $\mathrm{C}_{\mathrm{H}}=12$ at.\%. The transmission, $T$, and reflection, $R$, spectra were carried out between 400 and $2500 \mathrm{~nm}$ in steps of 
$2 \mathrm{~nm}$ using a computer-aided double-beam spectrophotometer (Shimadzu 3101 PC UV-VIS-NIR). The relative uncertainty in the transmittance and reflectance given by manufacturer is $0.2 \%$. Transmittance scans were performed using a glass substrate in the reference compartment of the same kind as the one used for the film deposition. The transmittance and reflectance were measured at the same incidence angle of $5^{\circ}$.

\section{Results and discussion}

\subsection{Evaluation of optical constants}

For the calculation of the optical constants, the refractive index $n$ and extinction coefficient $k$ were calculated using the envelope method suggested by Manifacier et al. [9] and modified by Swanepoel [10, 11]. The spectral envelopes of the transmittance, $T_{\max }(\lambda)$ and $T_{\min }(\lambda)$,.which are assumed to be continuous functions of the wavelength, were computed using a polynomial interpolation between extremes.

The transmittance spectra near the absorption edge and in the transparent region for the a-Si:H films is shown in Fig. (1). The computed envelope for the interference maxima is shown in the same figure as well. It can be observed that the thickness of films is almost uniform because the shrinking of the interference fringes is not remarkable. Considering the coherent interference of light inside the studied films, the optical constants, refractive index $(n)$ and extinction coefficient $(k)$, can by deduced from the transmittance (T) spectrogram using the Swanepoel procedure $[10,11]$.

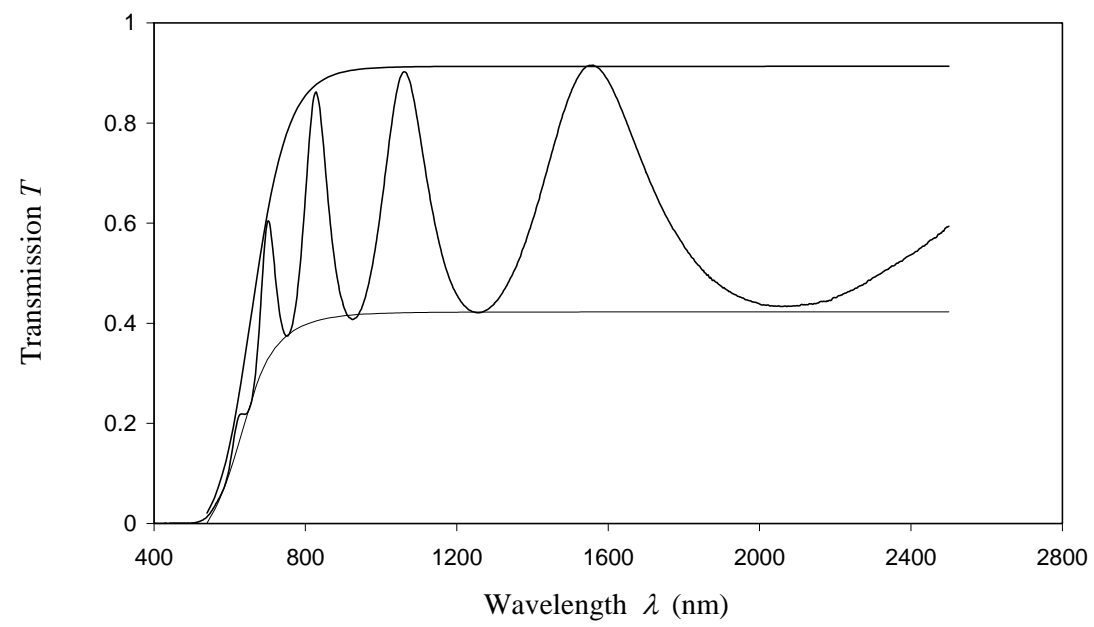

Fig. (1): The transmission spectrum for a-Si:H film along with the calculated envelope spectrum 
The change of the refractive index $n$ as a function of wavelength for different doping concentrations is shown in Fig. (2). It has been found that, the incorporation of $\mathrm{Al}^{3+}$ notably decreases the refractive index of the films. The same behavior was previously reported using boron as a dopant [12]. The estimated error for the refractive index, $\delta n$, is 0.003 .

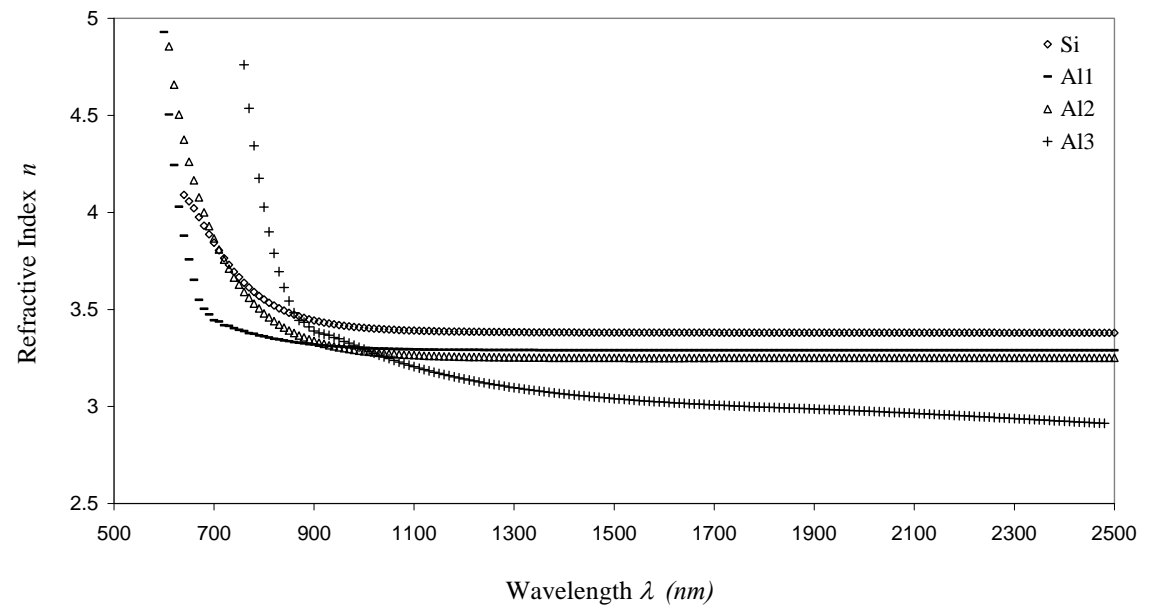

Fig. (2): The change of the refractive index $n$ versus wavelength for different doping concentrations

\subsection{Calculation of the Coordination Numbers}

To calculate the coordination number, the complete network approach was used according to the following equation [13]:

$$
\langle r\rangle=\frac{1}{N} \sum_{r \geq 1} r N_{r}
$$

where

$$
N=\sum_{r \geq 1} N_{r}
$$

and $N_{r}$ is the total number of atoms with $r$ bonds. The values of the calculated coordination numbers are given in Table (1). From the table, the decrease of the coordination number with doping could be seen. The reduction of the coordination number causes reduction of the mechanical hardness of the material as predicted by the rigidity percolation theory [13]. The expected percolation threshold for ideal glass $\langle\mathrm{r}\rangle=2.4$. Similar behavior was found in a$\mathrm{SiO}: \mathrm{H}$ films doped with boron [13]. The decrease in the coordination number with doping could attribute to the decrease in the refractive index [14]. 
Table 1: The doping concentration, coordination number, oscillation energy $E_{0}$, dispersion energy $E_{d}$, lattice energy $E_{l}$, static refractive index $n_{0}$, the average interband oscillator wavelength $\lambda_{0}$ and the average oscillator strength $S_{0}$ for different evaluated films.

\begin{tabular}{ccccccccc}
\hline Sample & $\begin{array}{c}\text { Doping } \\
\text { Conc. } \\
\text { (at. \%) }\end{array}$ & $\begin{array}{c}\text { Coordination } \\
\text { number }\end{array}$ & $\begin{array}{c}\boldsymbol{E}_{\mathbf{0}} \\
(\mathbf{e V})\end{array}$ & $\begin{array}{c}\boldsymbol{E}_{\boldsymbol{d}} \\
\mathbf{( e V )}\end{array}$ & $\begin{array}{c}\boldsymbol{E}_{\boldsymbol{l}} \\
(\mathbf{e V})\end{array}$ & $n_{o}$ & $\begin{array}{c}\boldsymbol{\lambda}_{\mathbf{0}} \\
(\mathbf{n m})\end{array}$ & $\begin{array}{c}\boldsymbol{S}_{\mathbf{0}} \\
\times \mathbf{1 0}^{-5} \\
(\mathbf{n m} \\
\mathbf{2}^{-}\end{array}$ \\
\hline $\mathrm{Si}$ & 0 & 3.640 & 2.59 & 16.32 & 0.062 & 2.94 & 402 & 4.73 \\
$\mathrm{Al} 1$ & 2.4 & 3.616 & 2.33 & 10.17 & 0.042 & 3.03 & 378 & 5.74 \\
$\mathrm{Al} 2$ & 3.6 & 3.604 & 2.28 & 10.06 & 0.061 & 3.06 & 415 & 4.77 \\
$\mathrm{Al} 3$ & 8.3 & 3.557 & 1.82 & 7.49 & 0.520 & 3.23 & 359 & 7.32
\end{tabular}

\subsection{Analysis of energy gap and absorption coefficient}

The method developed by Swanepoel $[10,11]$ was used to calculate the absorption coefficient $\alpha$. Fig. (3) shows the change of $\alpha$ as a function of energy for different doping concentrations, where three characteristic regions are indicated. Region I corresponds to band-to-band absorption, region II originates from absorption processes involving band tail states, and region III arises from absorption via Si dangling bond defect states in the middle of the band-gap. It could be seen from Fig. (4) that the absorption edge shifts towards lower photon energies as the $\mathrm{Al}$ content increases. A pronounced broadening of the absorption edge is also observed. Similar behavior was reported when doping with $\mathrm{Al} \mathrm{[6]} \mathrm{and} \mathrm{diborane} \mathrm{[15].}$

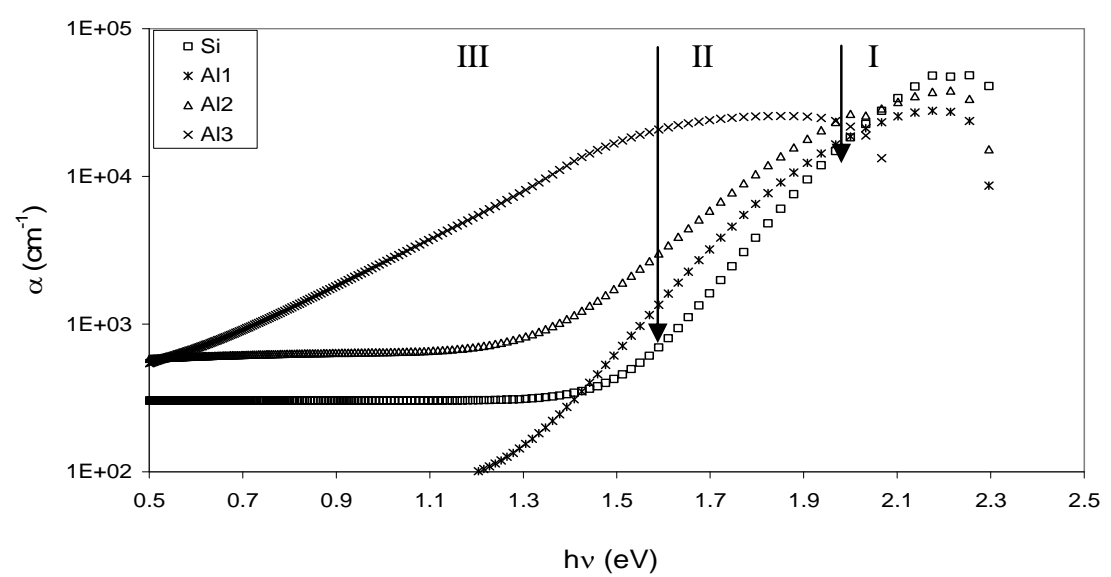

Fig. (3) : The absorption coefficient $\alpha\left(\mathrm{cm}^{-1}\right)$ as a function of the doping concentration. 


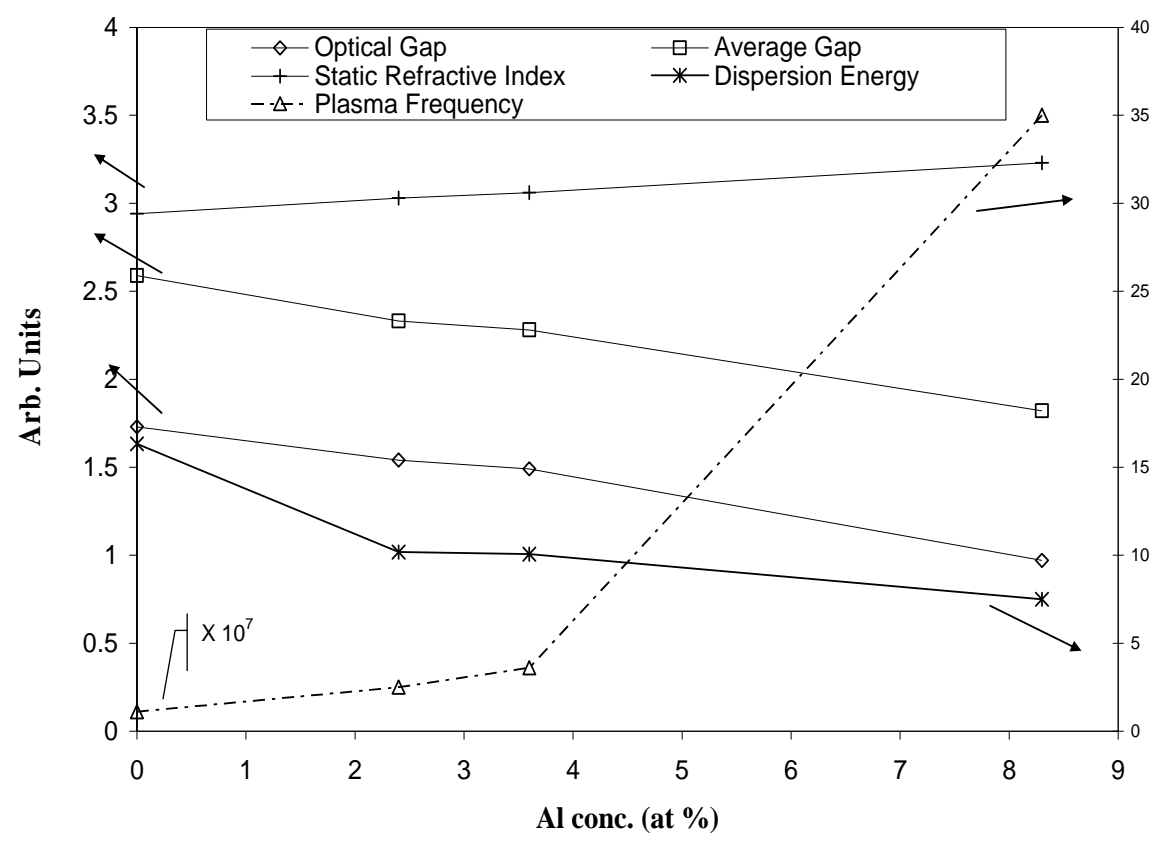

Fig (4): The change of average gap $E_{0}(\mathrm{eV})$, dispersion energy $E_{d}(\mathrm{eV})$, static refractive index $n_{o}$, indirect energy gaps $E_{g}^{\mathrm{opt}}(\mathrm{eV})$ and the plasma frequency $\omega_{p}\left(\mathrm{~s}^{-1}\right)$ with doping concentration for different evaluated films

An expression for the absorption coefficient, $\alpha(v)$, as a function of photon energy $(h v)$ for direct and indirect optical transitions is given by the following expression [16]

$$
\alpha(v)=\frac{A\left(h v-E_{g}^{o p t}\right)^{m}}{h v}
$$

where the exponent $m=1 / 2$ for allowed direct transition, while $m=2$ for allowed indirect transition. $E_{g}^{\text {opl }}$ is optical band gap energy and $A$ is a constant related to the extent of the band tailing. Plotting $(\alpha h v)^{1 / 2}$ against photon energy $(h v)$ gives a straight line with intercept equal to the optical energy band gap for indirect $\left(E_{g}^{\mathrm{opt}}\right)$ transitions as shown in Fig. (5). Table 2 lists the different determined energies, where the error for bandgap energies is \pm 0.003 . The value of the optical gap for the undoped sample agrees with previously reported values [6, 15, 17-19]. From Table (2), it could be seen that the optical band gap energy decrease with increasing the $\mathrm{Al}$ content. Similar results were previously reported [6, 15, 18-24]. This behavior was attributed to that the energy gap is 
determined by the weakest bond present in the alloy system ( $\mathrm{Si}-\mathrm{Al}$ or $\mathrm{Si}-\mathrm{B}$ ) which contributes to states near the band edges, lowering the Fermi level and consequently affecting the energy gap. Another reason was the increase of disorder in the network and broadening in the band tails produced by the introduction of large amount of threefold coordinated dopant atoms.

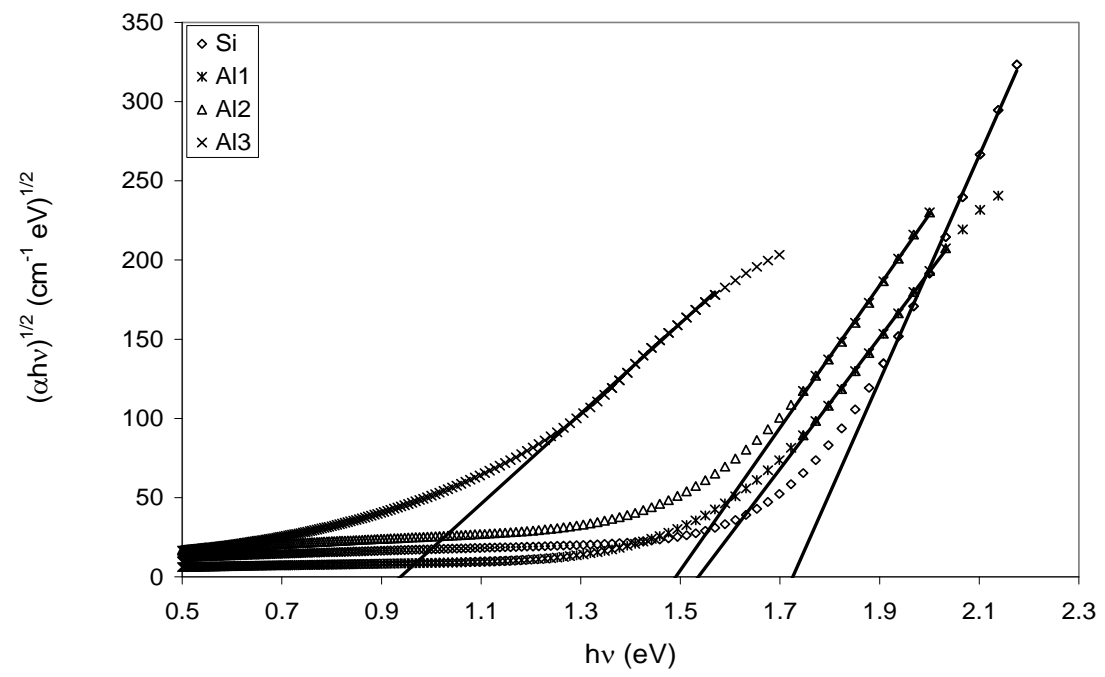

Fig. (5): Tauc plot to obtain the indirect energy gap for different doping concentrations.

Table 2: Indirect energy gaps $E_{g}^{\mathrm{op} 1}$, the Urbach energy $E_{\mathrm{U}}$, the ratio of free carrier concentration to the free carrier effective mass $N / \mathrm{m}^{*}$, the plasma frequency $\omega_{p}$ and the optical relaxation time $\tau$ for the used films.

\begin{tabular}{cccccc}
\hline Sample & $\begin{array}{c}E_{g}^{\mathrm{pl}} \\
\mathbf{( e V )}\end{array}$ & $\begin{array}{c}\boldsymbol{E}_{\mathbf{U}} \\
(\mathbf{m e V})\end{array}$ & $\begin{array}{c}\mathbf{N} / \mathbf{m}^{*} \\
\left(\mathbf{c m}^{-3}\right)\end{array}$ & $\begin{array}{c}\boldsymbol{\omega}_{\boldsymbol{p}} \\
\left(\mathbf{s}^{-1}\right)\end{array}$ & $\begin{array}{c}\boldsymbol{\tau} \times \mathbf{1 0}^{-8} \\
(\mathbf{s})\end{array}$ \\
\hline $\mathrm{Si}$ & 1.73 & 119 & $8.84 \times 10^{21}$ & $1.1 \times 10^{7}$ & 9.1 \\
$\mathrm{Al} 1$ & 1.54 & 124 & $4.90 \times 10^{22}$ & $2.5 \times 10^{7}$ & 3.2 \\
$\mathrm{Al} 2$ & 1.49 & 165 & $1.00 \times 10^{23}$ & $3.6 \times 10^{7}$ & 2.7 \\
$\mathrm{Al} 3$ & 0.97 & 262 & $6.10 \times 10^{23}$ & $3.5 \times 10^{8}$ & 0.29 \\
\hline
\end{tabular}

\subsection{Urbach energy and the tail of absorption edge}

It is well known that the shape of the fundamental absorption edge in the exponential (Urbach) region can yield information on the disorder effects [25]. With energy for the incident photon less than the band gap, the increase in absorption coefficient is followed with an exponential decay of density of states 
of the localized into the gap [26] and the absorption edge is known as Urbach edge. The lack of crystalline long-range order in amorphous/glassy materials is associated with a tailing of density of states [26]. At lower values of the absorption coefficient $\left(1 \mathrm{~cm}^{-1}<\alpha<10^{4} \mathrm{~cm}^{-1}\right)$, the extent of the exponential tail of the absorption edge characterized by the Urbach energy is given by [27].

$$
\alpha(h v)=\beta \exp \left(h v / E_{\mathrm{U}}\right)
$$

where $\beta$ is a constant, $E_{\mathrm{U}}$ is the Urbach energy which indicates the width of the band tails of the localized states. The optical absorption coefficient just below the absorption edge shows exponential variation with photon energy indicating the presence of Urbach's tail. Plotting $\ln (\alpha)$ vs. $h \alpha$ and taking the reciprocals of the slopes of the linear portion in the lower photon energy of these curves, $E_{\mathrm{U}}$ could be obtained where the error in computing $E_{\mathrm{U}}$ is \pm 0.001 .

The values of $E_{\mathrm{U}}$ for different compositions are listed in Table (2) and demonstrated in Fig. (6). As it can be seen, the addition of $\mathrm{Al}^{3+}$ ions is remarkably increasing the Urbach energy values, in agreement with others using different material for doping [21, 23]. Such increase could be related to the increase in the defect density due to the incorporation of threefold coordinated atoms and the spontaneous conversion of weak Si-Si bonds into dangling bonds [23]. The decrease in the optical gap, shown from Table (2), could be correlated to increase of disorder in the network, indicated by Urbach's energy $E_{U}$.

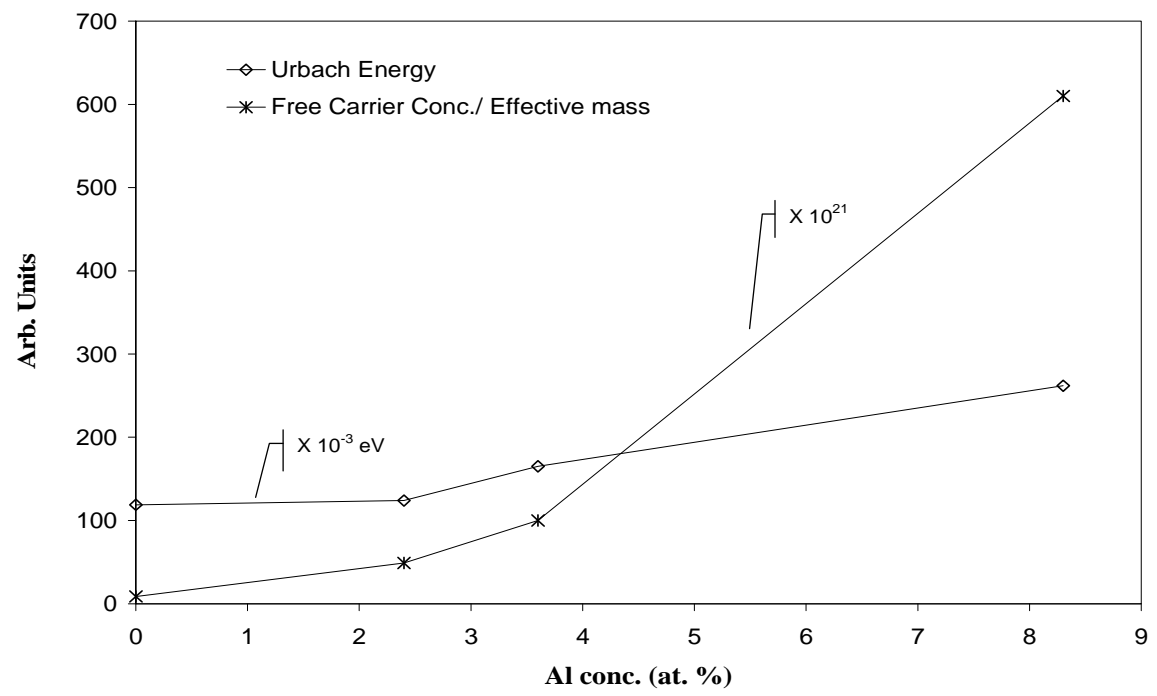

Fig. (6): The change of the Urbach energy $E_{\mathrm{U}}$ (mev) and the ratio of free carrier concentration to the free carrier effective mass $N / \mathrm{m}^{*}\left(\mathrm{~cm}^{-3}\right)$ with doping concentration for different evaluated films 


\subsection{Evaluation of material characteristic energies}

An interesting model (single oscillator model) describing the index dispersion behavior was proposed by Wemple and Di Domenico [28, 29]. This model is given to obtain a proper evaluation of the real part of the dielectric constant $\varepsilon_{r}(\omega)$. Hypotheses given to satisfy the model; (1) the important interband transitions can be approximated by individual oscillators $\omega_{n}$, (2) the summation over oscillators can be approximated, for $\omega<\omega_{n}$, by a summation of the first strong oscillator and a proper combination of the higher order contributions in which only the terms to order $\omega^{2}$ are retained providing a single effective oscillator model of $\varepsilon_{r}(\omega)$. It accounts for the dispersion curve according to the relation:

$$
n^{2}(\hbar \omega)=1+\frac{E_{d} E_{0}}{E_{0}{ }^{2}-(\hbar \omega)^{2}}
$$

where $\hbar \omega$ is the photon energy, $E_{0}$ and $E_{d}$ are two parameters connected to the optical properties of the material and they are known as the single oscillator energy and the oscillator strength (or dispersion energy) respectively. $E_{0}$ defines the average energy gap usually considered as the energy separation between the centers of both the conduction and the valence bands. $E_{d}$ is a measure of the average strength of the interband optical transitions and is related to the coordination number of the atoms. Plotting $\left(n^{2}-1\right)^{-1}$ against $(\hbar \omega)^{2}$, and fitting it to the straight part of the curve in the high-energy region allows obtaining from the slope and the intercept values of the single oscillator parameters ( $E_{0}$ and $\left.E_{\mathrm{d}}\right)$. Table 1 lists the obtained values of $E_{0}$ and $E_{\mathrm{d}}$ for the film compositions. The decrease in $E_{0}$ values obtained for the films are in accordance with the red shift in the edge of the recorded absorption spectra and the decrease in the optical band gap. As shown from table and demonstrated in Fig. (4), the average gap decreased with increase in doping concentration, same as previously reported conclusion using diborane for doping [15]. The decrease in values of $E_{d}$ could be attributed to the decrease in the average coordination number of the atoms.

In case of wavelengths much shorter than the phonon resonance, the lattice contribution is given from [30].

$$
n^{2}-1=E_{d} E_{0} /\left(E_{0}^{2}-E^{2}\right)-E_{l}^{2} / E^{2}
$$

where $E_{l}$ is the lattice energy. A plot of $\left(n^{2}-1\right)$ versus $1 / E^{2}$ approaches a straight line. At long wavelengths where $E^{2}<<E_{0}^{2}$ Eq. (5) can take the form [31]

$$
n^{2}-1=E_{d} / E_{0}-E_{l}^{2} / E^{2}
$$

The intercept is the ratio $E_{d} / E_{0}$, and the slope is $-E_{l}^{2}$. Thus the obtained values of $E_{l}$ are listed in Table 1. 


\subsection{Determination of inherent absorption wavelength and oscillator strength}

Many semiconductors materials have generally an inherent absorption in the ultraviolet range. The inherent wavelength of the ultraviolet absorption or the average interband oscillator wavelength, $\lambda_{0}$, and the average oscillator strength, $S_{0}$, is determined using Drude-Voigt dispersion formula as [32].

$$
n^{2}-1=\left(e^{2} / \pi m c^{2}\right) N_{1} S_{0} /\left(1 / \lambda_{0}^{2}-1 / \lambda^{2}\right)
$$

where $N_{1}$ is the number of molecules per unit volume of the film. Eq. (7) could be expressed in a single term Sellmeier oscillator with the refractive index as [33].

$$
\left(n_{o}^{2}-1\right) /\left(n^{2}-1\right)=1-\left(\lambda_{0} / \lambda\right)^{2}
$$

where $n_{o}$ is the static refractive index, which provides a good indication on the structure and density of the material, and it equals to $\sqrt{\varepsilon_{s t}}$ (static dielectric constant). $n_{o}$ and $\lambda_{0}$ can be evaluated from the plots of $\left(n^{2}-1\right)^{-1}$ versus $\lambda^{-2}$. Table 1 lists the obtained values of $n_{o}$ and $\lambda_{0}$. As shown form table, values of $n_{o}$ increased with increase in doping. The same behavior was previously reported when doping with boron $[15,20,21]$.

Furthermore, Eq. (8) can also take the form

$$
n^{2}-1=\left(S_{0} \lambda_{0}^{2}\right) /\left(1-\lambda_{0}^{2} / \lambda^{2}\right)
$$

where the average oscillator strength $S_{0}$ is defined as

$$
S_{0}=\left(n_{\infty}^{2}-1\right) / \lambda_{0}^{2}
$$

The computed values of $S_{0}$ are given in Table 1 .

The decrease in the average gap $E_{0}$ can be correlated with the increase in $n_{o}$ according to the formula [34]:

$$
n_{o}^{2}=1+A \frac{\left(\hbar \omega_{p}\right)^{2}}{E_{o}^{2}}
$$

where $\hbar \omega_{p}$ is the plasma energy for valence electrons and $A$ is a parameter depending on the matrix elements. As could be seen from Eq. (11), there exist 
an inversely proportional relationship between the average gap and the static refractive index, which is applied to the obtained data shown in Table (1).

\subsection{Complex dielectric constant and related characteristic parameters}

The complex dielectric function describes the interaction of electromagnetic waves with matter. The complex dielectric constant $\varepsilon$ of the material in terms of the optical constants $n$ and $k$ is given as:

$$
(n+i k)^{2}=\frac{\varepsilon}{\varepsilon_{0}}=\frac{\left(\varepsilon_{1}+i \varepsilon_{2}\right)}{\varepsilon_{0}}
$$

where $\varepsilon_{0}$ is the dielectric constant of free space. Separation of the real part and the imaginary one leads to the real part, [35].

$$
\varepsilon_{1}=n^{2}-k^{2}=\varepsilon_{\infty}-\frac{e^{2}}{4 \pi^{2} c^{2} \varepsilon_{0}} \frac{N}{m^{*}} \lambda^{2},
$$

and to the imaginary part,

$$
\varepsilon_{2}=2 n k=\frac{\varepsilon_{\infty} \omega_{p}^{2}}{8 \pi^{3} c^{3} \tau} \lambda^{3}
$$

where determination of the imaginary part of dielectric constant could be employed in determination of the optical relaxation time, $\tau$, of films. Here $\varepsilon_{0}$ is the free space dielectric constant, $N / \mathrm{m}^{*}$ the ratio of free carrier concentration, $N$, to the free carrier effective mass, $m^{*}$ and $\varepsilon_{\infty}$ is the high frequency dielectric constant. $\omega_{p}$ is the plasma frequency for the valence electrons and is given by:

$$
\omega_{p}=\left(e^{2} N / \varepsilon_{0} \cdot \varepsilon_{\infty} \cdot m^{*}\right)^{1 / 2}
$$

Using Eqs. (14)-(15) the parameters $N / m^{*}, \omega_{p}, \tau$ could be determined. Table 2 lists the evaluated values of the previous parameters. It is clear that both $\varepsilon_{o}$ and $N / m^{*}$ (Fig. (6)) change in the same manner with the average coordination number. Such unusual similarity in $\varepsilon_{o}$ and $N / \mathrm{m}^{*}$ behaviors has been observed for p-type Pb-Sn-Te chalcogenide system [36] and to Ge-Sb-Se system [37]. It may be attributed to the relatively higher carrier concentration that is consistent (assuming that $m^{*}$ is constant in the first approximation) with our case.

The $\varepsilon_{2}$ spectra plotted in Fig. (7) characterize the effect of doping $\mathrm{a}-\mathrm{Si}: \mathrm{H}$ films. As seen in figure, the entire curve is redshifted with increase in 
doping level. The same behavior was previously reported when using B with high doping levels [21].

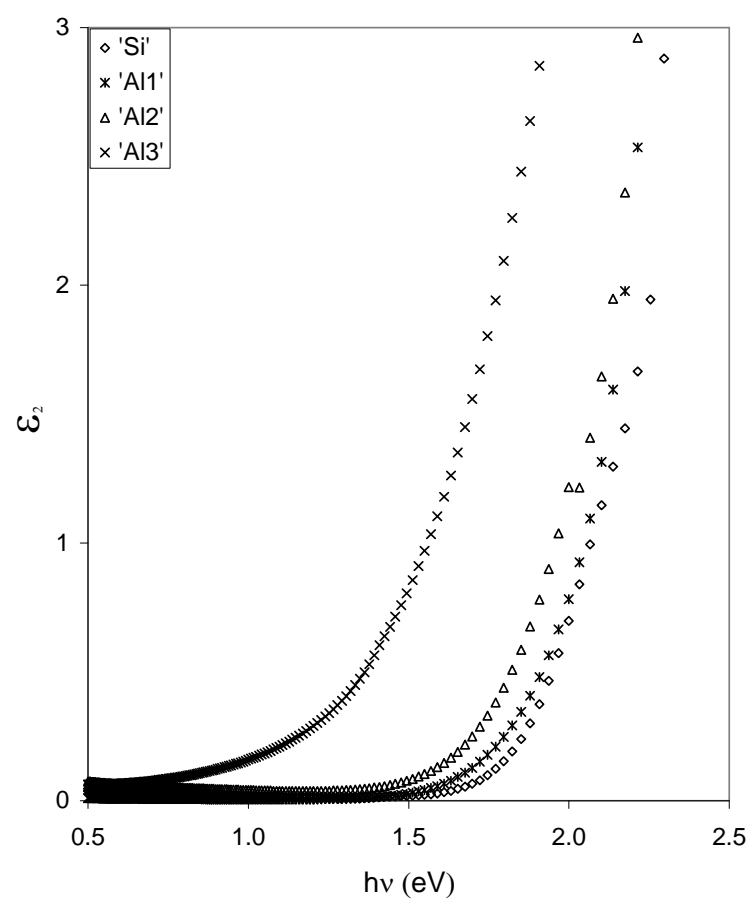

Fig. (7): The change of the imaginary part of the dielectric function $\varepsilon_{2}$ with doping.

Within the framework of the single oscillator model, the position of the maximum of $\varepsilon_{2}$ corresponds to the resonance energy $\hbar \omega_{o}$ of the oscillator. The resonance frequency $\omega_{o}$ is sensitive to the local chemical environment. The modification of this parameter is linked to the changes of the average separation between the valence band and the conduction band [28].

The static refractive index can be linked to the resonance frequency and the volume plasma frequency $[28,29]$ :

$$
n_{o}=n(\omega \rightarrow 0)=1-\frac{\omega_{p}^{2}}{\omega_{o}^{2}}
$$

The above equation suggests that $n_{0}$ is imaginary for $\omega_{0}<\omega_{p}$, i.e., the reflectivity of the material is $100 \%$. The plasma frequency depends on the 
density of the material. The redshift of $\varepsilon_{2}$ and the decrease of the optical gap with doping are compatible with a decrease of the resonance frequency of the oscillator. As a consequence, the increase of $n_{o}$ with doping is supported by an increase of $\omega_{p}$, as shown in Tables (1) and (2), and a decrease of $\omega_{o}$ in agreement with Eq. (16). The same behavior was previously reported [32].

\section{Conclusion:}

The inclusion of $\mathrm{Al}^{3+}$ in a-Si:H thin films caused a decrease in the refractive index values due to a decrease in the average coordination number of the system. A Decrease in dispersion energy values was also observed, indicating a strong ionic environment, which increase with increasing the aluminum concentration causing a decrease in the overall coordination number. The average gap decreases as well as the Tauc optical band gaps due to a decrease in the average bond energy of the system. An increase in Urbach energy indicating that more static disorder has taken place. An increase in free carrier concentration and a remarkable decrease in the relaxation time are obtained. An increase in the plasma energy and static refractive index is observed.

\section{References:}

1. D. E. Carlson, C. R. Wronski,. Appl. Phys. Lett., 28, 671 (1976).

2. J. Yang, A. Banerjee, K. Lord, S. Guha, Proceedings of the $2^{\text {nd }}$ World Conference on Photovoltaic Solar Energy Conversion, Vienna, Austria, p. 387 (1998)..

3. J. I Pankove, .Solar Cells, 24, 299 (1988).

4. J. I Pankove, "Semiconductors and semimetals", vol. 21B, Orland Academic Press, 1984.

5. A. Janotta, R. Janssen, M. Schmidt, T. Graf, L. Görgens, C. Hammerl, S. Schreiber, G. Dollinger, A. Bergmaier, B. Stritzker, and M. Stutzmann, $J$. Non-Cryst. Solids, 299-302, 579 (2002).

6. G. H. Lin, M. Kapur, J. O’M. Bockris, Sol. Energy Mater. Sol. Cell., 28, 29 (1992).

7. S. Tolansky, in : Multiple-Beam Interference.Microscopy of Metals, Academic, London, p.55 (1970).

8. A. K. Gosh, T. Mc Mahon, E. Rock, H. Wiesmann, J. Appl. Phys., 50, 3407 (1979).

9. J. C. Manifacier, J. Gasiot, J. P. Fillard, J. Phys. E:Sci. Instrum., 9, 1002 (1976).

10. R. .Swanepoel, J. Phys. E : Sci. Instrum., 16, 1214 (1983).

11. R. Swanepoel, J. Phys. E:Sci. Instrum., 17, 896 (1984).

12. M. H. Brodsky and P. A. Leary, J. Non-Cryst. Solids, 35\&36, 487 (1980). 
13. A. Janotta, R. Janssen, M. Schmidt, R. Graf, M. Stutzmann, L. Görgens, A. Bergmaier, G. Dollinger, C. Hammerl, S. Schreiber, and B. Stritzker, Phys. Rev. B69, 115206 (2004).

14. M. Abdel-Baki, F. A. Abdel-Wahab, A. Radi and F. El-Diasty, J. Phys. Chem. Solids, 68, 1457 (2007).

15. A. Lloret, Z. Y. Wu, M. L. Theye, I. El Zawawi, J. M. Siefert and B. Equer, Appl. Phys., A55, 573(1992).

16. E. A. Davis, N. F. Mott, Phil Mag., 22903 (1970)

17. P. Hess, Thin Solid Films, 241, 318 (1994).

18. D. Jousse, J. Said and J. C. Bruyere, Thin Solid Films, 124, 49 (1985).

19. S. Ray, P. Chaudhuri, A. K. Batabyal and A. K. Barua, Sol. Energy Mat. Sol. Cells, 10, 335 (1984).

20. V. Bursikova, P. Sladek, P. St'ahel, and J. Pursik, J. Non-Cryst. Solids, 352, 1238 (2006).

21. A. Hadjadi, P. St'ahel, P. Roca i Cabarrocas, V. Paret, Y. Bounouh, and J. C. Martin, J. Appl. Phys., 83, 830 (1998).

22. A. M. Perez, F. J. Renero, C. Zuniga, A. Torres and C. Santiago, J. Phys.: Condens. Matter, 17, 3975 (2005).

23. M. M. de Lima and F. C. Marques, J. non-cryst. sol, 299-302, 605 (2002).

24. G. Talukder, J. A. Cowan, D. E. Brodie and J. D. Leslie, Can. J. Phys., 62, 848 (1984)

25. G.D. Cody, T. Tiedje, B. Abeles, B. Brooks, Y. Goldstein, Phys. Rev. Lett., 47, 1480 (1981).

26. B. Abay, H. S. Guder, Y. K. Yogurtchu, Solid State. Commun., 112, 489 (1999).

27. M. A. Hassan, C. A. Hogarth, J. Mater. Sci., 23, 2500 (1988).

28. S. H. Wemple, M. Di Domenico, Phys. Rev., B3, 1338 (1971).

29. S. H. Wemple, Phys. Rev., B7, 3767 (1973).

30. S. H. Wemple, Appl. Opt. 18, 31 (1979).

31. H. Poignant, Electron. Lett. 17, 973 (1981).

32. S. Hirota, T. Izumitani, J. Non-Cryst. Solids 29, 109 (1978).

33. M. Abdel-Baki, F. A. Abdel Wahab, F. El-Diasty, Mater. Chem. Phys. 96, 201 (2006).

34. D. R. Penn, Phys. Rev., B128, 2093 (1962).

35. T. S. Moss, G. J. Burrell, E. Ellis, Semiconductor Opto-Electronics, Butterworths, London, (1973)

36. V. Gopal, Infrared Phys. 20, 161 (1980)

37. M. M. Wakked, E. Kh. Shoker, S. H. Mohamed, J. Non-Cryst. Solids 265, 157 (2000) 